\title{
IBS for RHIC operation below transition energy and various RF systems
}

\author{
A. Fedotov
}

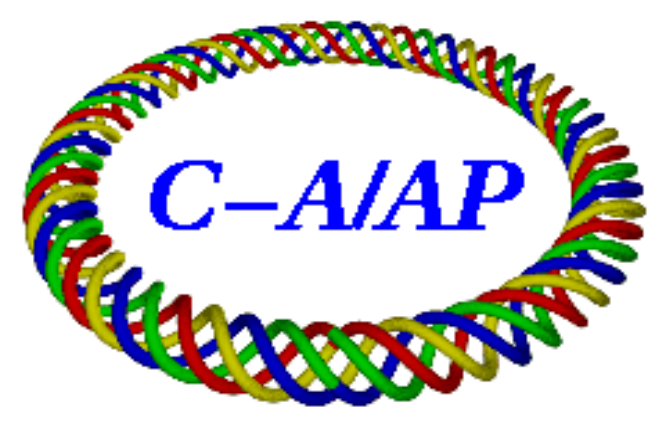

\section{Collider-Accelerator Department Brookhaven National Laboratory \\ Upton, NY 11973}

Notice: This document has been authorized by employees of Brookhaven Science Associates, LLC under Contract No. DE-AC02-98CH10886 with the U.S. Department of Energy. The United States Government retains a nonexclusive, paid-up, irrevocable, world-wide license to publish or reproduce the published form of this document, or allow others to do so, for United States Government purposes. 


\title{
IBS FOR RHIC OPERATION BELOW TRANSITION ENERGY AND VARIOUS RF SYSTEMS
}

\author{
Alexei Fedotov \\ C-AD, Brookhaven National Laboratory
}

\section{Introduction}

Following first successful runs in RHIC at low energies for physics (Beam Energy Scan I) there is a strong interest in further low-energy RHIC operation in the range of $\gamma=4-10$ but with higher luminosity (Beam Energy Scan II). An electron cooling was proposed to increase luminosity at these low energies [1-2]. Both transverse and longitudinal Intra-beam Scattering (IBS) can be counteracted with electron cooling. In addition, the phase-space density of the hadron beams can be further increased by providing stronger cooling. Unfortunately, the defining intensity limitation for low energies in RHIC is expected to be the space charge [3]. Experimental studies of the spacecharge effects and beam lifetime in RHIC were reported in Refs. [4-5]. Additional limitations at low energies and their possible mitigations were discussed in Refs. [6-7].

In Ref. [8] it was suggested that better luminosity performance may be expected by using lower frequency RF system and longer bunches compared to the baseline $28 \mathrm{MHz} \mathrm{RF}$, which is being used for RHIC operation with heavy ions. Recently, even lower frequency RF of $4.5 \mathrm{MHz}$ was proposed for future low-energy RHIC operation [9].

Although low-frequency RF systems in RHIC (either 9 or $4.5 \mathrm{MHz}$ ) seem to address possible limitations of beam lifetime observed at low energies, the resulting ion beam temperatures in beam rest frame get further from an equilibrium which lead to a much stronger longitudinal IBS. This would put stronger requirement on electron cooling system under consideration. However, operation below transition energy allows us to exploit an IBS feature that drives the transverse and longitudinal beam temperatures towards equilibrium by minimizing the longitudinal diffusion rate using larger longitudinal beam emittance and larger RF voltage. Such studies were performed before to explore IBS redistribution at low energies for the 28 and $56 \mathrm{MHz}$ RHIC RF systems [10]. This Note presents results of similar studies for newly proposed 4.5 and $9 \mathrm{MHz}$ RF systems.

\section{IBS redistribution below transition energy}

\section{$28 \mathrm{MHz}$ RF system}

Presently low-energy RHIC operation employs $28 \mathrm{MHz}$ RF system. The maximum possible total RF gap voltage on two cavities is now $500 \mathrm{kV}$ (the use of $450 \mathrm{kV}$ with beam operation was demonstrated). For previous low-energy RHIC operation, ion beams were produced using the Tandems injector resulting in a relatively small longitudinal emittances delivered to RHIC. The values of the longitudinal emittance values measured in 2010 are shown in Table 1.

\begin{tabular}{|l|l|}
\hline$\gamma$ & $\mathrm{S}_{95 \%}, \mathrm{eV}-\mathrm{s} /$ nucleon \\
\hline 4.1 & 0.25 \\
\hline 6.2 & 0.33 \\
\hline 10 & 0.36 \\
\hline
\end{tabular}

Table 1. Longitudinal emittance was measured at AGS extraction in 2010 by P. Harvey. 
Recently, heavy ion operation switched from the Tandems to the EBIS injector. To achieve high bunch intensities comparable to those provided previously with the Tandems several RF merges were employed in the Booster and AGS in 2012 [11]. As a result, longitudinal emittance at RHIC injection was larger than in previous runs based on the Tandem with the longitudinal emittance of $S=0.6 \mathrm{eV}-\mathrm{s} / \mathrm{n}$ for the bunch intensity of $\mathrm{N}=1.6 \cdot 10^{9}$. Some modest improvement in the longitudinal emittance can be expected in the future with a better execution of the RF mergers [11]. For low-energy RHIC operation where we are limited by the space-charge effects, the highest bunch intensity is not required and some RF merges can be dropped which should result in smaller longitudinal emittance. Thus, we assume that for bunch intensities of about $\mathrm{N}=0.8 \cdot 10^{9}$ and $0.4 \cdot 10^{9}$ we can expect the longitudinal emittance of around 0.3 and $0.15 \mathrm{eV}-\mathrm{s} / \mathrm{n}$, respectively.

To take into account space-charge limitation in RHIC, for discussion in this note, we use such bunch intensity that the incoherent space-charge tune spread $\Delta \mathrm{Q}_{\mathrm{sc}}$ does not exceed the value of $\Delta \mathrm{Q}_{\mathrm{sc}}=0.05$. A discussion of the space-charge limit in a collider and corresponding beam lifetime will be reported elsewhere. Table 2 shows IBS growth rates with present $28 \mathrm{MHz}$ RF system for ion beam at $\gamma=4$ keeping the space-charge tune spread constant at $\Delta Q_{\mathrm{sc}}=0.05$ by changing ion bunch intensity. One can see that longitudinal IBS growth rate strongly increases when momentum spread becomes smaller and beam temperatures get further from an equilibrium. More RF voltage can be applied which would result in larger momentum spread. However, shorter bunch length would require further decrease of bunch intensity to keep the space-charge tune spread constant thus reducing luminosity.

\begin{tabular}{|l|l|l|l|l|l|}
\hline $\begin{array}{l}\mathrm{S}_{95 \%}, \\
\mathrm{eV} \text {-s/nucleon }\end{array}$ & $\sigma_{\mathrm{p}}$ & $\sigma_{\mathrm{s}}, \mathrm{m}$ & $\mathrm{N}, \times 10^{8}$ & $\begin{array}{l}\text { transverse IBS } \\
\tau_{\mathrm{x}}^{-1}, \mathrm{sec}^{-1}\end{array}$ & $\begin{array}{l}\text { longitudinal IBS } \\
\tau_{\mathrm{z}}^{-1}, \mathrm{sec}^{-1}\end{array}$ \\
\hline 0.3 & 0.00053 & 2.4 & 8 & 0.001 & 0.001 \\
\hline 0.2 & 0.00044 & 2.0 & 7 & 0.00075 & 0.003 \\
\hline 0.15 & 0.00037 & 1.7 & 6 & 0.0005 & 0.006 \\
\hline
\end{tabular}

Table 2. IBS growth rates $\left(\tau_{\mathrm{x}}{ }^{-1}=\mathrm{d} \varepsilon_{\mathrm{x}} / \varepsilon_{\mathrm{x}} \mathrm{dt}, \tau_{\mathrm{z}}{ }^{-1}=\mathrm{d} \sigma_{\mathrm{p}}{ }^{2} / \sigma_{\mathrm{p}}{ }^{2} \mathrm{dt}\right)$ for different longitudinal momentum spread (beam temperature) for the $28 \mathrm{MHz}$ RF with total gap voltage of $150 \mathrm{kV}, \gamma=4.1$ and transverse beam emittance of $\varepsilon=15 \mu \mathrm{m}$ (95\%, normalized).

In this Note, we use convention of $95 \%$ longitudinal emittance $S$ defined as:

$$
S_{95 \%}=6 \pi \frac{m c^{2}}{c}(\beta \gamma) \sigma_{p} \sigma_{z}
$$

where $m$ is the rest mass per nucleon, $\sigma_{z}$ is rms bunch length and $\sigma_{p}$ is rms momentum spread.

\section{Small momentum spread and longitudinal heating due to IBS}

Choosing lower frequency RF system and thus longer bunches will reduce space-charge tune spread value. This will allow to cool beam transversely which in turn should allow us to reduce beta-function at the interaction point resulting in higher luminosity [8]. In addition, transverse cooling will enable larger transverse acceptance which should provide better beam lifetime for the same space-charge tune spread value, as observed experimentally [4-5]. However, longer ion bunch will have smaller momentum spread and thus stronger longitudinal IBS growth rates.

Below transition energy IBS drives the transverse and longitudinal beam temperatures towards equilibrium (in a smooth-lattice approximation). This suggests that longitudinal heating can be stopped (at least initially) if the longitudinal beam temperature is close or larger than transverse. 
If there is enough RF voltage, one can increase longitudinal beam temperature by shrinking the bunch length or perhaps inject the beam with larger longitudinal emittance (provided that RF bucket acceptance is sufficiently large). By doing so, one can redistribute IBS between the longitudinal and transverse degrees of freedom and minimize losses from the RF bucket due to the longitudinal IBS [10].

If longitudinal beam temperature is smaller than transverse, the longitudinal heating rate (diffusion coefficient) can be approximated by:

$$
\frac{d \sigma_{p}^{2}}{d t}=\frac{r_{i}^{2} c N_{i} \Lambda_{c}}{8 \beta^{3} \gamma^{3} \varepsilon_{x, u n}^{3 / 2}\left\langle\beta_{x}^{1 / 2}\right\rangle \sigma_{s}}\left(1-\sqrt{\frac{\Delta_{z}}{\Delta_{x}}}\right),
$$

where $r_{\mathrm{i}}$ is the classical radius of ion, $N_{\mathrm{i}}$ is number of ions in the bunch, $\Lambda_{\mathrm{c}}$ is the Coulomb logarithm, $\sigma_{\mathrm{s}}$ is the RMS bunch length, $\sigma_{\mathrm{p}}$ is the RMS momentum spread, $\beta_{\mathrm{x}} \approx \beta_{\mathrm{y}}$ is the average beta-function around the ring, $\mathcal{E}_{\mathrm{x}} \approx \mathcal{E}_{\mathrm{y}}$ is the transverse beam emittance (rms, un-normalized), and $\Delta_{z}$ and $\Delta_{x}$ are the longitudinal and transverse rms velocity spread of ion beam in beam rest frame, respectively. An expression in Eq. (2) roughly describes the longitudinal heating rate when $\Delta_{z} / \Delta_{x}<1$. It is valid only for the case of smooth-lattice approximation and round beam and is similar to the relaxation coefficient for anisotropic plasma (in such a regime with the longitudinal heating one gets transverse cooling in the other plane). In the limit of very small longitudinal velocity spread when $\Delta_{z} / \Delta_{x}$ goes to zero one recovers longitudinal diffusion coefficient typically used for the high-energy approximation [12]. More accurate expressions for such diffusion coefficient with anisotropic velocity distribution can be found, for example, in Refs. [13-15]. Here we use approximate scaling in Eq. (2) for illustrative purpose only to demonstrate why one gets very strong longitudinal IBS growth rate when beam temperatures deviate from equilibrium with decreasing longitudinal momentum spread. In the Tables in this Note we show results of numerical simulations of IBS growth rates with the BETACOOL code [16] using realistic RHIC lattice without approximations.

Below we summarise results of IBS redistribution study and required RF voltage for the proposed low-frequency RF systems of 9 and $4.5 \mathrm{MHz}$.

\section{$9 \mathrm{MHz}$ RF system}

For the $9 \mathrm{MHz} \mathrm{RF}$, the longitudinal emittance $S=0.15 \mathrm{eV}-\mathrm{s} / \mathrm{n}$ and bunch intensity $\mathrm{N}=5 \cdot 10^{8}$ resulting IBS growth rates are shown in Table 3. Small initial values of the space-charge tune spread indicate that cooling of transverse emittance by at least a factor of two should be possible, which will increase transverse acceptance and should improve beam lifetime.

\begin{tabular}{|l|l|l|l|l|l|}
\hline $\begin{array}{l}\text { RF Voltage, } \\
\mathrm{kV}\end{array}$ & $\sigma_{\mathrm{p}}$ & $\sigma_{\mathrm{s}}, \mathrm{m}$ & $\Delta \mathrm{Q}_{\mathrm{sc}}$ & $\begin{array}{l}\text { transverse IBS } \\
\tau_{\mathrm{x}}^{-1}, \mathrm{sec}^{-1}\end{array}$ & $\begin{array}{l}\text { longitudinal IBS } \\
\tau_{\mathrm{z}}^{-1}, \mathrm{sec}^{-1}\end{array}$ \\
\hline 25 & 0.00018 & 3.6 & 0.02 & -0.00036 & 0.023 \\
\hline 50 & 0.00021 & 3.0 & 0.024 & -0.00029 & 0.018 \\
\hline 100 & 0.00026 & 2.5 & 0.03 & -0.00011 & 0.011 \\
\hline
\end{tabular}

Table 3. IBS rates $\left(\tau_{\mathrm{x}}{ }^{-1}=\mathrm{d} \varepsilon_{\mathrm{x}} / \varepsilon_{\mathrm{x}} \mathrm{dt}, \tau_{\mathrm{z}}{ }^{-1}=\mathrm{d} \sigma_{\mathrm{p}}{ }^{2} /{\sigma_{\mathrm{p}}}^{2} \mathrm{dt}\right)$ for $S=0.15 \mathrm{eV}-\mathrm{s} / \mathrm{n}$, bunch intensity $\mathrm{N}=5 \mathrm{e} 8$ for the 9 $\mathrm{MHz}$ RF, $\gamma=4.1$ and transverse beam emittance of $\varepsilon=15 \mu \mathrm{m}$ (95\%, normalized).

One can see that even with $100 \mathrm{kV}$ RF voltage longitudinal IBS growth times are very short which requires very fast cooling to counteract such IBS. Requirement on electron cooling can be 
relaxed if we can operate with larger longitudinal emittance which also requires larger RF acceptance and thus RF voltage. This is shown in Table 4 for several longitudinal emittances.

\begin{tabular}{|l|l|l|l|l|l|l|l|}
\hline $\begin{array}{l}\mathrm{S}_{95}, \\
\mathrm{eV}-\mathrm{s} / \mathrm{n}\end{array}$ & $\begin{array}{l}\mathrm{RF} \text { bucket } \\
\text { acceptance } \\
\mathrm{A}_{\mathrm{s}}, \mathrm{eV}-\mathrm{s} / \mathrm{n}\end{array}$ & $\begin{array}{l}\mathrm{RF}, \\
\mathrm{kV}\end{array}$ & $\sigma_{\mathrm{p}}$ & $\sigma_{\mathrm{s}}, \mathrm{m}$ & $\Delta \mathrm{Q}_{\mathrm{sc}}$ & $\begin{array}{l}\text { transverse IBS } \\
\tau_{\mathrm{x}}^{-1}, \mathrm{sec}^{-1}\end{array}$ & $\begin{array}{l}\text { longitudinal IBS } \\
\tau_{\mathrm{z}}^{-1}, \mathrm{sec}^{-1}\end{array}$ \\
\hline 0.3 & 0.5 & 100 & 0.00036 & 3.6 & 0.02 & 0.00017 & 0.0027 \\
\hline 0.3 & 0.6 & 150 & 0.0004 & 3.24 & 0.022 & 0.00027 & 0.002 \\
\hline 0.4 & 0.7 & 200 & 0.00049 & 3.5 & 0.021 & 0.0004 & 0.0008 \\
\hline
\end{tabular}

Table 4. IBS rates $\left(\tau_{\mathrm{x}}{ }^{-1}=\mathrm{d} \varepsilon_{\mathrm{x}} / \varepsilon_{\mathrm{x}} \mathrm{dt}, \tau_{\mathrm{z}}{ }^{-1}=\mathrm{d} \sigma_{\mathrm{p}}{ }^{2} /{\sigma_{\mathrm{p}}}^{2} \mathrm{dt}\right)$ for $S=0.15 \mathrm{eV}-\mathrm{s} / \mathrm{n}$, bunch intensity $\mathrm{N}=5 \mathrm{e} 8$ for the $9 \mathrm{MHz}$ RF, $\gamma=4.1$ and transverse beam emittance of $\varepsilon=15 \mu \mathrm{m}$ (95\%, normalized).

From Table 4 one can see that longitudinal IBS growth rate can be strongly minimized with large longitudinal emittance of $S=0.4 \mathrm{eV}-\mathrm{s} / \mathrm{n}$ which also requires large RF voltage of about $200 \mathrm{kV}$. For the RF voltage of $150 \mathrm{kV}$ one can already achieve reasonably weak IBS growth rates. The IBSdriven luminosity lifetime is shown in Figs. 1-2 for parameters from Table 4. With electron cooling such IBS-driven luminosity lifetime could be strongly improved.

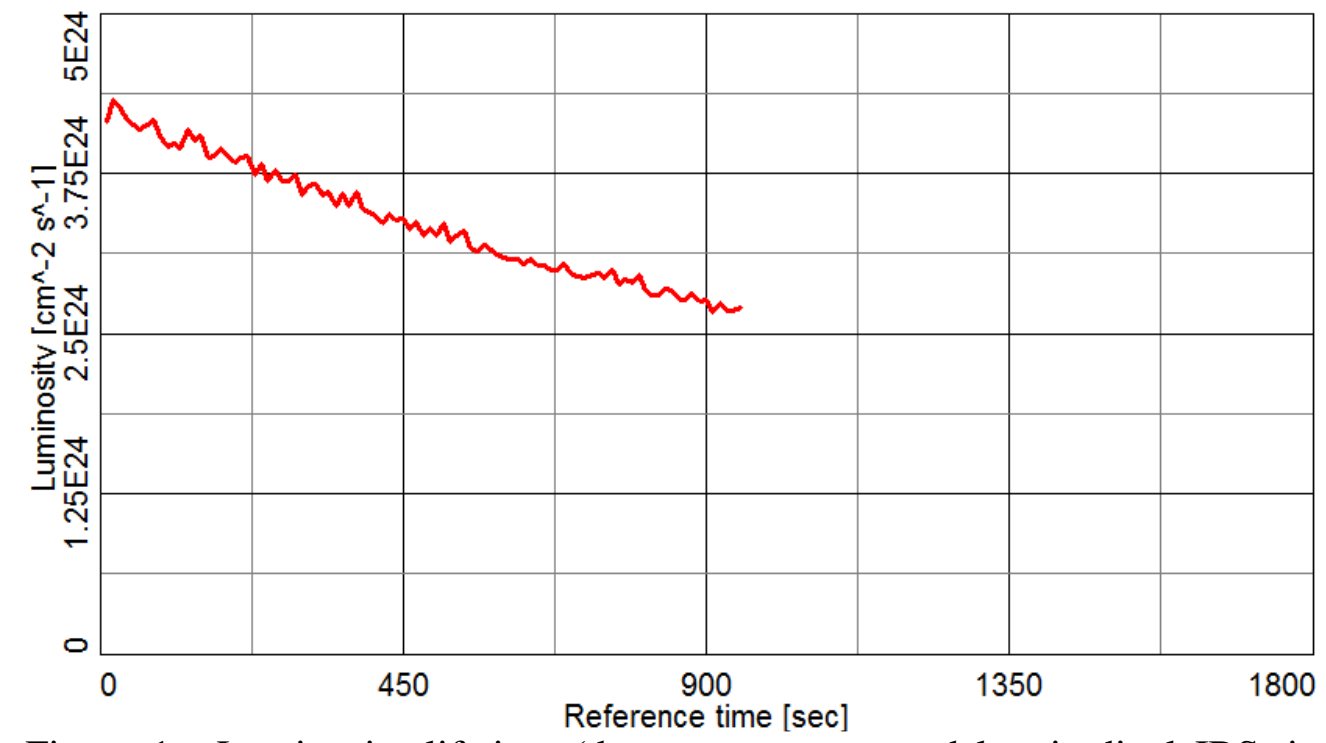

Figure 1. Luminosity lifetime (due to transverse and longitudinal IBS, including particle losses from the RF bucket) at $\gamma=4.1$, for initial beam parameters in Table 4 with the $200 \mathrm{kV}$ of $9 \mathrm{MHz} \mathrm{RF}$ and longitudinal emittance $S=0.4 \mathrm{eV}$-s/n. 


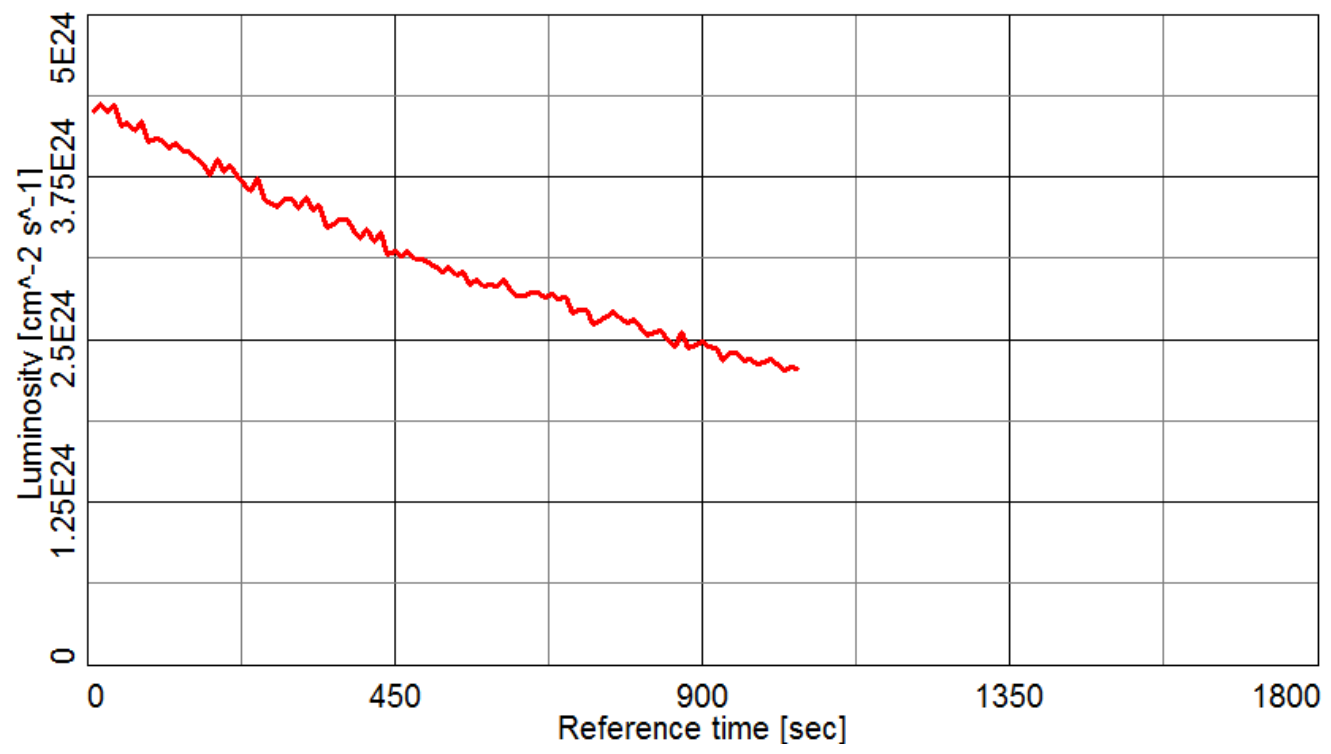

Figure 2. Luminosity lifetime (due to transverse and longitudinal IBS) at $\gamma=4.1$, for initial beam parameters in Table 4 with $150 \mathrm{kV}$ of $9 \mathrm{MHz} \mathrm{RF}$ and $S=0.3 \mathrm{eV}-\mathrm{s} / \mathrm{n}$.

It appears that about $150 \mathrm{kV}$ of the $\mathrm{RF}$ voltage is required for the $9 \mathrm{MHz}$ system to make longitudinal IBS growth rate reasonably small. For RF voltage of $100 \mathrm{kV}$, an acceptance of the RF bucket is not sufficient for making use of large longitudinal emittance to control IBS growth rates.

\subsection{MHz RF system}

Since 4.5 MHz RF system corresponds to a harmonic $\mathrm{h}=60$ of the particle revolution frequency in RHIC, we can have only 60 colliding bunches in each ring. To provide the same initial luminosity as for the $9 \mathrm{MHz}$ RF with 120 bunches, intensity per bunch should be increased for the 4.5 MHz RF. Below we use bunch intensity of $\mathrm{N}=7.5 \cdot 10^{8}$ for the $4.5 \mathrm{MHz}$ compared to $\mathrm{N}=5 \cdot 10^{8}$ for the $9 \mathrm{MHz}$ RF to compensate for the number of bunches and longer bunch length, compared to the 9 $\mathrm{MHz}$ RF.

\begin{tabular}{|l|l|l|l|l|l|l|l|}
\hline $\begin{array}{l}\mathrm{S}_{95}, \\
\mathrm{eV}-\mathrm{s} / \mathrm{n}\end{array}$ & $\begin{array}{l}\mathrm{RF} \text { bucket } \\
\text { acceptance } \\
\mathrm{A}_{\mathrm{s}}, \mathrm{eV}-\mathrm{s} / \mathrm{n}\end{array}$ & $\begin{array}{l}\mathrm{RF} \\
\text { Voltage, } \\
\mathrm{kV}\end{array}$ & $\sigma_{\mathrm{p}}$ & $\sigma_{\mathrm{s}}, \mathrm{m}$ & $\Delta \mathrm{Q}_{\mathrm{sc}}$ & $\begin{array}{l}\text { transverse IBS } \\
\tau_{\mathrm{x}}{ }^{-1}, \mathrm{sec}^{-1}\end{array}$ & $\begin{array}{l}\text { longitudinal IBS } \\
\tau_{\mathrm{z}}{ }^{-1}, \mathrm{sec}^{-1}\end{array}$ \\
\hline 0.15 & 0.46 & 10 & 0.00012 & 5.35 & 0.02 & -0.0007 & 0.064 \\
\hline 0.4 & 0.8 & 30 & 0.00026 & 6.6 & 0.016 & -0.00006 & 0.006 \\
\hline 0.4 & 1.0 & 50 & 0.00029 & 5.8 & 0.019 & 0.00001 & 0.005 \\
\hline 0.5 & 1.3 & 80 & 0.00036 & 5.8 & 0.019 & 0.00016 & 0.0026 \\
\hline
\end{tabular}

Table 5. IBS rates $\left(\tau_{\mathrm{x}}^{-1}=\mathrm{d} \varepsilon_{\mathrm{x}} / \varepsilon_{\mathrm{x}} \mathrm{dt}, \tau_{\mathrm{z}}{ }^{-1}=\mathrm{d} \sigma_{\mathrm{p}}{ }^{2} / \sigma_{\mathrm{p}}{ }^{2} \mathrm{dt}\right)$ for bunch intensity of $\mathrm{N}=7.5 \mathrm{e} 8$ for $4.5 \mathrm{MHz} \mathrm{RF}$, $\gamma=4.1$ and transverse beam emittance of $\varepsilon=15 \mu \mathrm{m}$ (95\%, normalized).

From Table 5 one can see that relatively large longitudinal emittance and RF voltage are required to minimize longitudinal IBS rates and achieve luminosity lifetime comparable to the one with the 9 $\mathrm{MHz}$ RF system. Figure 3 shows luminosity lifetime (mostly due to longitudinal IBS and losses from the RF bucket) for the longitudinal emittance of $S=0.5 \mathrm{eV}-\mathrm{s} / \mathrm{n}$ and $\mathrm{RF}$ voltage of $80 \mathrm{kV}$ for the 4.5 MHz system. 


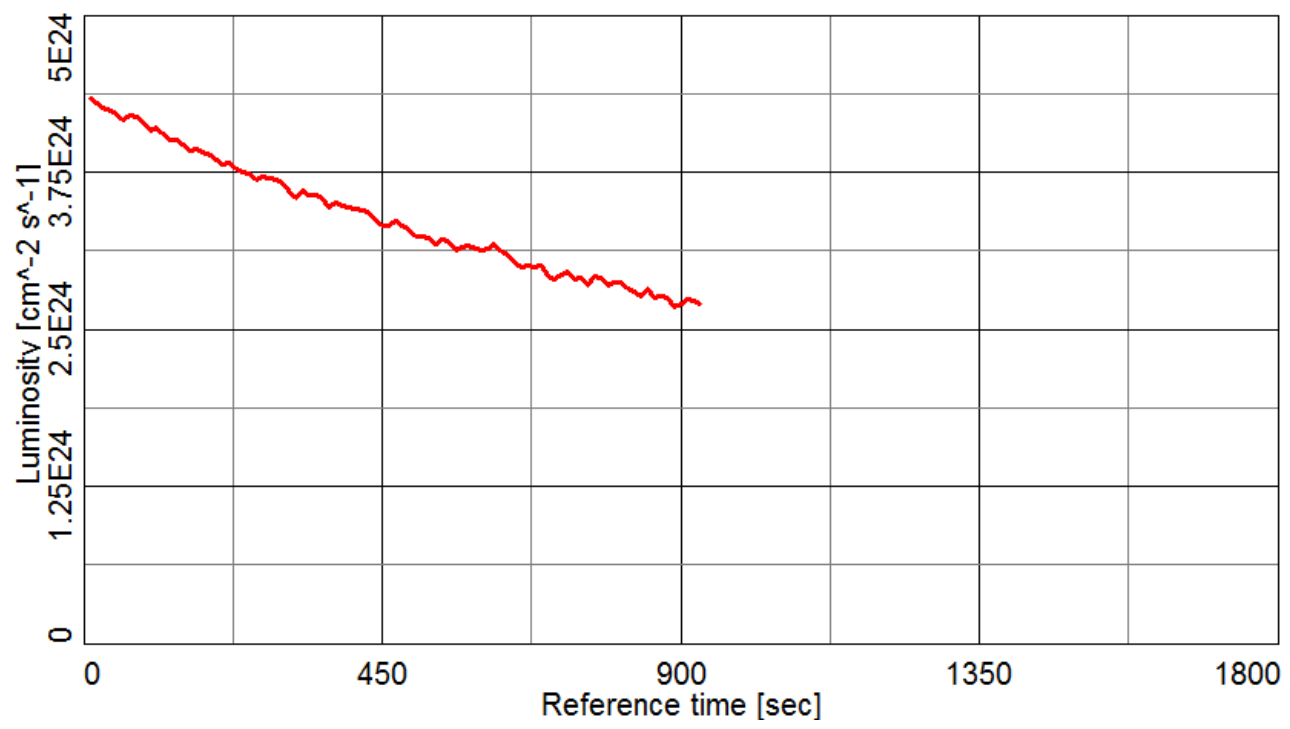

Figure 3. Luminosity lifetime (due to transverse and longitudinal IBS) at $\gamma=4.1$, for initial beam parameters in Table 5 with $80 \mathrm{kV}$ RF voltage for the $4.5 \mathrm{MHz}$ system and $S=0.5 \mathrm{eV}-\mathrm{s} / \mathrm{n}$.

\section{Summary}

Low-frequency RF systems in RHIC (either 9 or $4.5 \mathrm{MHz}$ ) seem to offer possible remedies for some beam lifetime limitations observed at low energies in RHIC. However, for long ion bunches the resulting ion beam temperatures in beam rest frame get further from an equilibrium which leads to a strong longitudinal IBS. This can be mitigated to some extent by using larger longitudinal emittance and RF voltage.

In simulations, a reasonably good luminosity lifetime due to IBS and comparable luminosities were obtained by using large value of longitudinal emittance which in turn requires large RF voltage of about $150 \mathrm{kV}$ and $80 \mathrm{kV}$ for the 9 and $4.5 \mathrm{MHz} \mathrm{RF}$, respectively. Any of these new RF systems with such voltages seem to be a good choice for RHIC operation with longer bunches and electron cooling.

Electron cooling should improve luminosity for these scenarios significantly by counteracting longitudinal IBS and by providing cooling of transverse emittance which in turn should allow us to reduce the beta-function at the interaction point.

\section{Acknowledgement}

We would like to thank Vladimir Litvinenko and Alex Zaltsman for many useful discussions on this subject. In simulations, we used BETACOOL code developed at JINR, Dubna, Russia.

\section{References}

[1] A. Fedotov, I. Ben-Zvi, X. Chang, D. Kayran, T. Satogata, Proc. of COOL07 (Bad Kreuznach, Germany, 2007), p. 243.

[2] A. Fedotov, I. Ben-Zvi, X. Chang, D. Kayran, V. Litvinenko, E. Pozdeyev and T. Satogata, BNL Collider-Accelerator Department Tech Note: C-A/AP/307 (April 2008). 
[3] A. Fedotov, I. Ben-Zvi, X. Chang, D. Kayran, V. Litvinenko, E. Pozdeyev, T. Satogata, Proc. of HB2008 workshop (Nashville, TN 2008), p. 75, WGA10 (2008).

[4] A. Fedotov, M. Blaskiewicz, W. Fischer, T. Satogata, S. Tepikian, Proc. of HB2010 workshop (Morschah, Switzerland, 2010), p. 634, THO1C03 (2010).

[5] A. Fedotov, M. Bai, M. Blaskiewicz, W. Fischer, D. Kayran, C. Montag, S. Tepikian, G. Wang and T. Satogata, Proc. of PAC'11 (New York, NY), p. 2285, THP081 (2011).

[6] C. Montag, BNL Collider-Accelerator Department Tech Note: C-A/AP/421 (January 2011).

[7] C. Montag et al., BNL C-AD Tech Note: C-A/AP/435 (October 2011).

[8] A. Fedotov and M. Blaskiewicz, "Potential for luminosity improvement for low-energy RHIC operation with long bunches", BNL C-AD Tech Note: C-A/AP/449 (February 2012).

[9] V. Litvinenko, "Choosing RF system for low energy RHIC operation", BNL C-AD Tech Note: C-A/AP/476 (December 2012).

[10] A. Fedotov, "IBS and potential luminosity improvement for RHIC operation below transition energy", BNL C-AD Tech Note: C-A/AP/339 (December 2008).

[11] C. Gardner, "Longitudinal aspects of beam in the RHIC injectors", BNL Collider-Accelerator Department Seminar (September 2012).

[12] A. Fedotov, BNL C-AD Tech Note: C-A/AP/168 (September 2004).

[13] S. Ichimaru and M.N. Rosenbluth, Phys. Fluids 13, p. 2778 (1970).

[14] S. Nagaitsev, Phys. Rev. STAB 8, 064403 (2005).

[15] J. Wei, Proc. of PAC'93, p. 3651 (1993).

[16] BETACOOL code: http://betacool.jinr.ru. 